\title{
An automatic experimental apparatus to study arm reaching in New World monkeys
}

\author{
Allen Yin $^{\mathrm{a}, \mathrm{b} *}$, Jehi An ${ }^{\mathrm{a}, \mathrm{b}, \mathrm{e}_{*}}$, Gary Lehew ${ }^{\mathrm{b} *}$, Mikhail A. Lebedev ${ }^{\mathrm{a}, \mathrm{b}}$, Miguel A. L. Nicolelis ${ }^{\mathrm{a}, \mathrm{b}, \mathrm{c}, \mathrm{d} \dagger}$ \\ ${ }^{a}$ Biomedical Engineering, Duke University, Durham, NC, USA \\ ${ }^{\mathrm{b}}$ Duke Center for Neuroengineering, Duke University, Durham, NC, USA \\ ${ }^{\mathrm{c}}$ Department of Neurobiology, Duke University Medical Center, Durham, NC, USA \\ ${ }^{\mathrm{d}}$ Edmond and Lily Safra International Institute of Neuroscience of Natal, Natal, Brazil. \\ * The three authors contributed equally for this study.
}

${ }^{\dagger}$ Corresponding author:

Miguel A. L. Nicolelis

Box 3209 Dept. of Neurobiology

Duke University

Durham, NC 27710

nicoleli@neuro.duke.edu

Office 1+ 9196844580

Fax 1+919668 0734 


\section{Abstract}

Background: Several species of the New World monkeys have been used as experimental models in biomedical and neurophysiological research. However, a method for controlled arm reaching tasks has not been developed for these species.

New Method: We have developed a fully automated, pneumatically driven, portable, and reconfigurable experimental apparatus for arm-reaching tasks suitable for these small primates.

Results: We have utilized the apparatus to train two owl monkeys in a visually-cued arm-reaching task. Analysis of neural recordings demonstrates directional tuning of the M1 neurons.

Comparison with Existing Method(s): Our apparatus allows automated control, freeing the experimenter from manual experiments.

Conclusion: The presented apparatus provides a valuable tool for conducting neurophysiological research on New World monkeys.

\section{Keywords}

New World monkeys, Animal model, Behavior, Neural recordings, Arm reaching, Experimental control 


\section{Introduction}

New world monkeys include a diverse group of five families of primates of Parvorder Platyrrhini, primarily living in the tropical forests of South America. These primates are small to midsized ranging from the pygmy marmoset, at 14 to $16 \mathrm{~cm}$ and a weight of 120 to 190 grams to the southern muriqui, at 55 to $70 \mathrm{~cm}$ and a weight of 12 to $15 \mathrm{~kg}$. Several species of Platyrrhini have been studied in biomedical research including squirrel monkeys (Error! Hyperlink reference not valid.; Nudo and Milliken 1996; Nudo, Milliken et al. 1996), the common marmoset (Mansfield 2003; Takemoto, Izumi et al. 2011), and owl monkeys (Allman and Kaas 1971; Merzenich, Kaas et al. 1983; Kaas 1987; Nicolelis, Ghazanfar et al. 1998; Wessberg, Stambaugh et al. 2000; Bao, Chang et al. 2004). The nocturnal owl monkey (Aotus Trivirgatus) has been of particular interest to neurophysiological study. It has a relatively smooth cortex which facilitates mapping (Allman and Kaas 1971 ; Kaas 1987) and allows implantation of a large number of microwires in multiple cortical areas (Nicolelis, Ghazanfar et al. 1998). Further, chronic cortical implants of microwire arrays in owl monkeys have been shown to maintain good neuronal recording quality for at least 3-5 years (Sandler $\underline{2005})$.

Arm reaching tasks have been of particular interest in neurophysiological research, used often to study neural encoding of movement (Georgopoulos, Schwartz et al. 1986; Georgopoulos, Kettner et al. 1988; Kettner, Schwartz et al. 1988; Schwartz, Kettner et al. 1988; McIntyre, Stratta et al. 1998; Lebedev and Wise 2001), visuo-motor systems (Flash and Henis 1991; Wise, Di Pellegrino et al. 1992; Lacquaniti and Caminiti 1998; Batista, Buneo et al. 1999), and most recently brain-machine interfaces (BMIs) (Error! Hyperlink reference not valid.; Carmena, Lebedev et al. 2003; Scherberger, Goodale et al. 2003; Lebedev and Nicolelis 2006; Scherberger and Andersen 2007; O'Doherty, Lebedev et al. 2011; Ifft, Shokur et al. 2013). Most of these studies, with a few exceptions (Error! Hyperlink reference not valid.; Fitzsimmons, Drake et al. 2007), have been performed on rhesus monkeys, resulting in a host of experimental apparatuses targeted toward this species (Error! Hyperlink reference not valid.; Mitz, Boring et al. 2001; Mitz 2005; Wilson, Kim et al. 2005). Yet, a thorough method for executing arm reaching experiments with New World monkeys has not been sufficiently developed.

Since New World monkeys present an attractive experimental model for neurophysiological recording in behaving primates, we sought to build an experimental apparatus for arm-reaching tasks suitable for this small primate species. Such an apparatus needs to be fully automated, include natural reinforcement methods for training the monkey, convenient ways to manipulate attention and detect monkey movements, and produce low electrical noise in neural recording experiments. The fully automated, portable apparatus presented here addressed these requirements by integrating a customizable pellet feeder, visual stimuli, and pneumatically controlled actuators. This device operated successfully in training owl monkeys to perform visually-cued reach tasks and later in intra-cortically stimulated reach tasks and may be applied to a variety of neurophysiological and BMI studies.

\section{Materials and Methods}

\subsection{Operation Overview}

The device (Figure 1) consists of three doors, three pellet dispensers, and three traps assembled onto a metal barrier, which is mounted on a linear slider, totaling 10 degrees of freedom. Each degree of freedom is driven by a pneumatic cylinder (20-40 psi) to eliminate electrical noise in neural recording. In an experiment, pellets are dispensed into the traps and then cleared away. If the metal barrier is at a forward position on the slider close to the monkey, and the door corresponding to that trap is open, then the monkey can obtain the reward. 


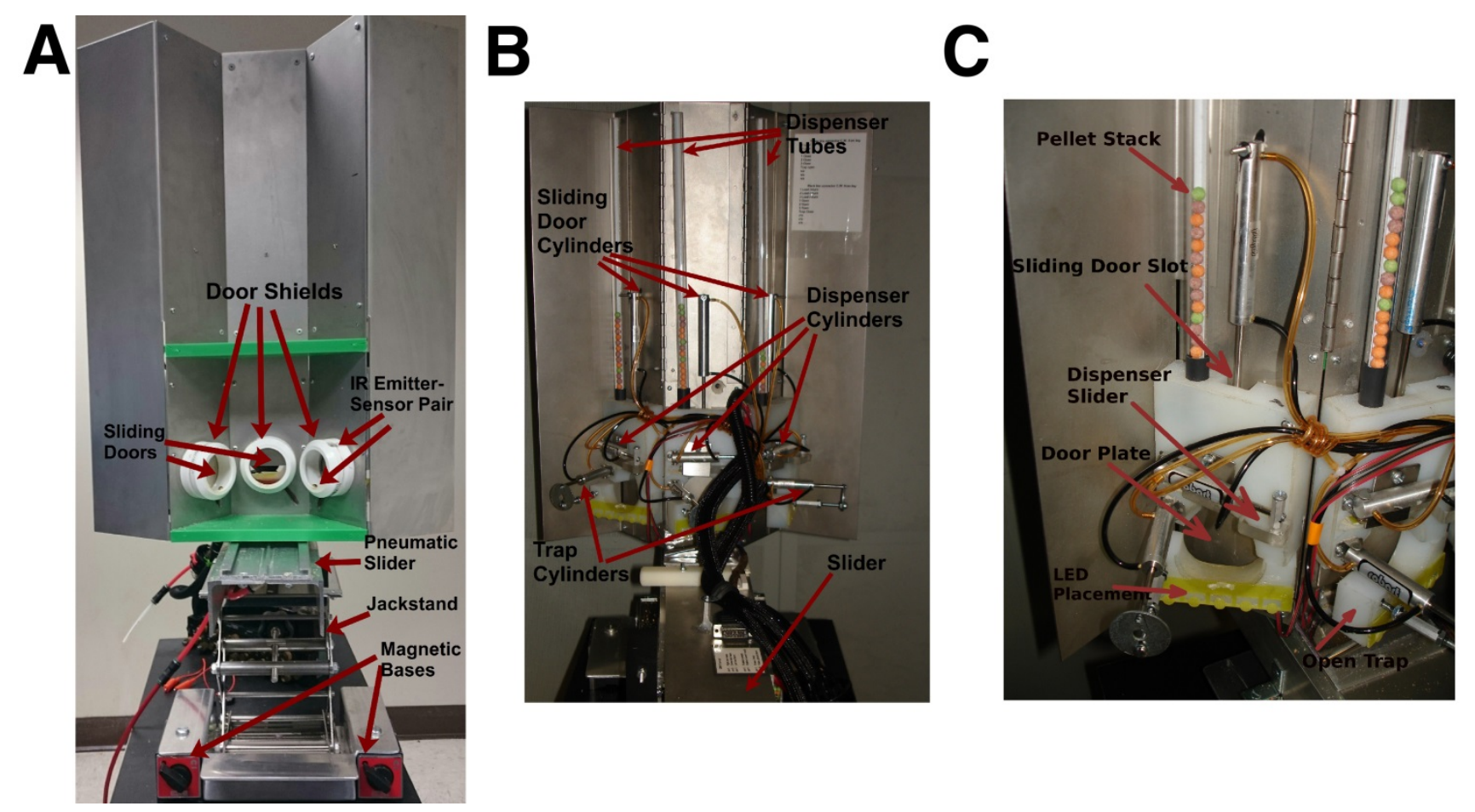

Figure 1. Experimental apparatus. (A) Front view of the experimental apparatus with the jack stand raised. In the figure, the middle sliding door is half lowered. (B) Back of the apparatus. (C) A detailed view of the pellet dispenser and the door assembly. Both traps in the picture are open while the sliding doors are closed. The LED is not shown but its placement is labeled.

\subsection{Pneumatics}

A manifold of eight 4-way two-position solenoid air valves (Numatics 226-432B) is used to control the air cylinders. The trap cylinders are controlled by a single air valve, whereas every other cylinder has a valve dedicated to it alone. A 24V DC-power supply powers the manifold, and a single source of compressed air is connected to the input of manifold.

An array of 8 optoisolators (IXYS LCA710) mounted on a custom printed circuit board (PCB) is used to electronically control the valves. An optoisolator acts as a switch opening or closing the connection between the $24 \mathrm{~V}$ power supply and a valve, depending on its input signal. Closing the connection activates the corresponding valve, changing the direction of air flow through it. The optoisolators take $5 \mathrm{~V}$ signals as inputs, allowing them to easily interface to the GPIO pins of many micro-controllers (Arduino, Trinket 5V, Pinguino, Raspberry Pi, etc) as well as the National Instrument Data Acquisition (NIDAQ) system. Indicator LEDs at the input pins of the optoisolators light up when the corresponding valve is activated.

The pneumatic air cylinders are double-acting: they have air pressure ports on each end of the cylinder which force a plunger to either extend or retract by applying pressure to one port while venting the other in a controlled manner. The speed of application and venting of the pressure establishes the speed of the plunger movement. A rod is attached to the plunger that serves to transmit the motion to the mechanism it drives.

\subsection{Base Slider}


The linear slider consists of a rectangular aluminum plate $(50 \mathrm{~cm} \times 11 \mathrm{~cm})$ mounted on a slider carriage riding on a modified box rail. The box rail itself is mounted onto the back side of a half-square tube (see Figure 2A). The double-acting cylinder is bolted onto the bottom side of the half-square tube with its rod attached to the back of the aluminum plate via an angle bracket.

A barrier made from five metal panels $(10 \mathrm{~cm}$ wide, $50 \mathrm{~cm}$ high) is mounted on top of slider, which serves as a base to assemble the other components of the system. As the rod of the slider cylinder extends or retracts, the slider along with the metal barrier, slides along the rails. The cylinder plunger is extended by default and the barrier is approximately $35 \mathrm{~cm}$ away from a monkey seated in front of the device. Air pressure and venting rate can be adjusted to select the slider speed and smoothness.

This entire setup is attached on top of a jack-stand that is bolted onto a square bracket base. The platform can be raised to a height of $50 \mathrm{~cm}$, allowing easy adjustments of the device for different experimental setups and monkey height. Each of the two side flaps of the bracket base is attached to the top of a magnetic base. Thus this apparatus is portable and can be mounted onto any metal surface.

\subsection{Doors}

On the bottom of the three center panel sections of the metal barrier are round holes $(4 \mathrm{~cm}$ diameter) through which a monkey can reach for pellet treats. The three center sections are arranged in a trapezoidal shape so they are equidistant from a monkey seated facing front-center of the barrier. A short round section of PVC ( $3 \mathrm{~cm}$ length, $4 \mathrm{~cm}$ diameter) shield is glued onto each opening. Air cylinders with the rods attached to aluminum plates $(7 \mathrm{~cm} \times 9 \mathrm{~cm})$ are mounted vertically on the back of the barrier right above the holes. Activation of the cylinders would push the aluminum plate doors down to cover the corresponding holes.

Blocks of $9 \mathrm{~cm} \times 14 \mathrm{~cm} \times 3 \mathrm{~cm}$ (width $\times$ height $\times$ thickness) ultra-high-molecular-density plastic (UHMD) are bolted onto the back of the three center sections covering the sliding doors (see Figure 2B). Slots are cut on the top side of the blocks against the barrier to let the door plate slide through. Holes of $4 \mathrm{~cm}$ in diameter are also cut in the blocks corresponding to the locations of the barrier holes to be used as reward traps.

A

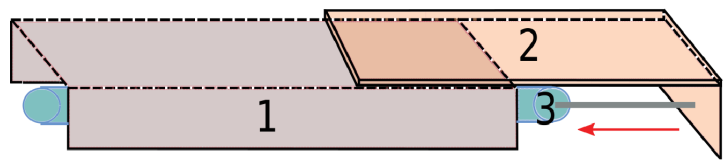

C

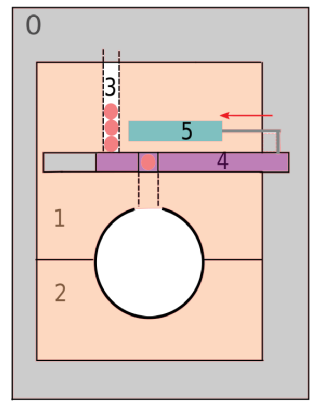

D

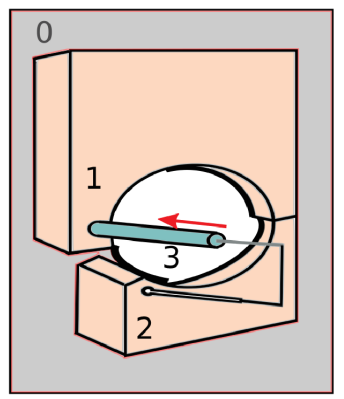

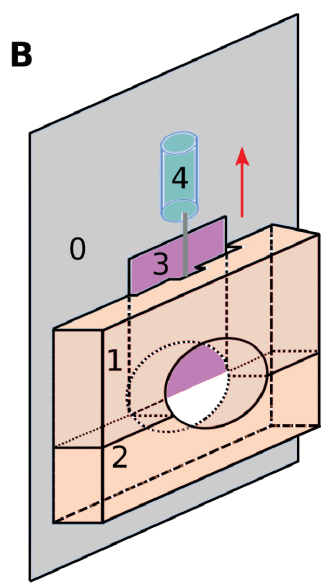


Figure 2. Simplified drawing of device mechanisms (not to scale). Red arrows indicate retraction of pneumatic plunger. (A) Base Slider. In order of numbering: half-square tube, slider carriage/box rail assembly, pneumatic slider. (B) Sliding Door. In order of numbering: back of metal barrier, top UHMD section, trap, sliding door, pneumatic cylinder. (C) Pellet Dispenser viewed from back of the apparatus. In order of numbering: back of metal barrier, top UHMD section, trap, pellet dispenser tube, horizontal slider, pneumatic cylinder. (D) Trap. In order of numbering: back of metal barrier, top ABS section, trap in opening position, pneumatic cylinder.

\subsection{Pellet Dispenser}

In our previous experiments, owl monkeys were well motivated by food rewards (Fitzsimmons, Drake et al. 2007; Fitzsimmons, An et al. 2009). For this reason, we incorporated a solid food dispenser in this system.

The pellet dispenser consists of Plexiglas tubes of $1 \mathrm{~cm}$ in diameter mounted vertically on the back of the metal barrier, with pneumatically controlled gates that allow only one treat to be dispensed at a time. The pellets (0.26-inch Bio-Serv Dustless Precision Pellets for primates) are stacked on top of each other inside the tubes.

The dispenser tubes are inserted off-center into the top of the UHMD blocks. Horizontal channels of $1 \mathrm{~cm}$ are cut across the blocks right above their hole cutouts. Pieces of $8 \mathrm{~cm} \mathrm{x} 1 \mathrm{~cm} \mathrm{x} 3 \mathrm{~cm}$ (width $\mathrm{x}$ height $\mathrm{x}$ thickness) sliders are inserted into these channels, with holes of $0.5 \mathrm{~cm}$ in diameter. Air cylinders are mounted on the UHMD blocks with the rods attached to one end of the sliders. A vertical channel is drilled from the center of the horizontal channel through the door cutout in the UHMD block to let the pellet drop down into the trap (see Figure 2C).

When the cylinder rod is fully retracted, the slider fits inside the horizontal channel completely, and the hole inside the slider is directly below the dispenser tube, accepting only the bottom-most pellet from the dispenser tube. When the cylinder rod is fully extended, the slider is pulled out such that its hole and the single pellet within are pulled to the middle of the horizontal channel. The pellet then drops through the vertical channel to a rest on top of the trap. The monkey can then reach through the open door-hole to access the treat.

\subsection{Trap}

Traps are used to control the pellet dispensing process. The rectangular UHMD block is cut horizontally through the axis of its door cutout and the bottom piece serves as a trap. Only one side of the trap remains attached to the back of the metal barrier via a hinge, allowing the other end to rotate away from the plane of the barrier. An air cylinder is attached to the top UHMD piece via another hinge perpendicular to the barrier, directly above the free end of the trap. The cylinder is coupled to a bolt on the free end of the trap such that the extension of the rod pushes the trap away from the barrier, while retraction brings it parallel to the barrier (see Figure 2D).

In the closed position, where the cylinder rod is retracted, the trap will catch any pellet deposited by the corresponding dispenser. When the trap is rotated away in the open position, any existing pellets will roll to the floor and it will not be able to catch any more pellets. All three trap cylinders are connected to the same air valve so they open and close collectively.

\subsection{Sensors}

Infrared (IR) emitter-sensor pairs are mounted on the inside of the PVC shields in front of the doors to detect the monkey's reaching hand. The emitter is placed diametrically across from the sensor so the monkey's hand interrupts the IR beam. Both the emitter and the sensor are powered by a $5 \mathrm{~V}$ 
power supply, and the sensor output can be sampled by NIDAQ or custom microcontroller digital inputs.

\subsection{Light Stimuli}

Light emitting diodes (LEDs) are attached to the back of the traps (Figure 1C) pointing upward to illuminate their corresponding door-holes. We limited the current through the LEDs to less than $20 \mathrm{~mA}$ by connecting resistors to it in series so that the LEDs can be directly controlled by a NIDAQ without using the optoisolators to source the required current. If not using the NIDAQ, the current limit is constrained by the total current that the custom micro-controllers can source out of their I/O pins.

\subsection{Control System and Software}

In our experiment, a customized PC (AMD Opteron processor $2.4 \mathrm{GHz}$ with $2 \mathrm{~GB}$ of RAM) installed with Microsoft Windows XP was used to control the device. We have explored two ways for the PC to interface with the device.

First, the PC communicated to the device through a NIDAQ PCI card (PCI-6220) and connected to a connector block (NI SCB-68). The optoisolators' input signals were active low and were controlled by the DAQ's digital output pins. The IR sensor outputs were connected to the DAQ's digital input pins. The LEDs were connected to the DAQ's digital output pins. Custom Matlab scripts were used to control the outputs to and record the inputs from the NIDAQ system. The advantages of this approach are the short installation time and short signal delay. The main disadvantages are the cost of the National Instrument hardware and its compatibility with non-Windows systems. The experiments described here used this approach for the short installation time.

The second method used an Arduino Uno micro-controller. The PC communicated with the Arduino through USB2.0. The Arduino's digital I/O pins served as the optoisolators' and LEDs' input control signals, and received the IR sensors' output. Custom Arduino software were used to monitor the state of the pins and communicated with Python 2.7 scripts on the PC for experimental control and datarecording.

Note that while many off-the-shelf micro-controllers are capable of such interface, we explored using the Arduino for ease of use, low price, and abundant community. The advantages of this approach are the order of magnitude hardware cost reduction, wide platform support, and high level of control protocol customization. The main disadvantages are the long setup time as custom software must be written for the Adruino and the PC, and the longer, more variable signal delay through USB2.0.

\subsection{Subjects}

All surgical and behavioral procedures conformed to the National Research Council Guide for the Care and Use of Laboratory Animals and were approved by the Institutional Animal Care and Use Committee.

Two owl monkeys, monkey A (female, 8 years old) and monkey P (female, 2 years old) were trained on a visually-cued arm reaching task. Experimental sessions were conducted during weekdays. To avoid hypoglycemia in the controlled food paradigm, animals were fed twice each weekday. They were given a small amount in the morning and then the majority of their ration in the afternoon. The morning ration was decreased to keep the monkey motivated during the experiments. After they returned to their home cages, the animals were given monkey chow and fruit. Daily records were kept of the amount of chow and fruit given. Any subject losing more than $15 \%$ of its ad libitum weight was temporarily removed from the study and given free access to chow, fruit, and jelly. In our experiments, 
both monkeys maintained stable body weight. We continually monitored the behavior and appearance of the animals for signs of stress or healthy issues. No such signs were observed.

On the weekends, the monkeys received fruit and chow ad libitum. On all days, the monkeys were given free access to water in their home cages.

Monkey P was not implanted. Monkey A had microwire electrode arrays chronically implanted (Error! Hyperlink reference not valid.): 4 x 8 arrays in the left primary motor (M1) and left posterior parietal (PP) cortices, and $2 \times 8$ arrays in both the left and right primary somatosensory (S1) cortices. The chronic electrode assemblies were prepared in-house from $50 \mu \mathrm{m}$ Teflon-coated steel microwires, paced $300 \mu \mathrm{m}$ apart.

\subsection{Behavioral Task}

Before the naïve monkeys were introduced to the task of interest, they were first trained to reach through the open doors for pellets dropped at random, with no regard to number of attempts. Once they learned where to obtain treats, the slider $\mathrm{m}$ between reaches, with speed adjusted to not scare the monkeys. After the monkeys became accustomed to this set up, light stimulus was added. The LED corresponding to the pellet location was turned on indefinitely until the monkeys reached for the correct door and obtained the treat. After about 5 to 10 such experimental sessions, the monkeys were then started on the visually-cued experimental task training. During these preliminary sessions, the monkeys were constrained to the chair for a progressively longer time, up to 2 hours.

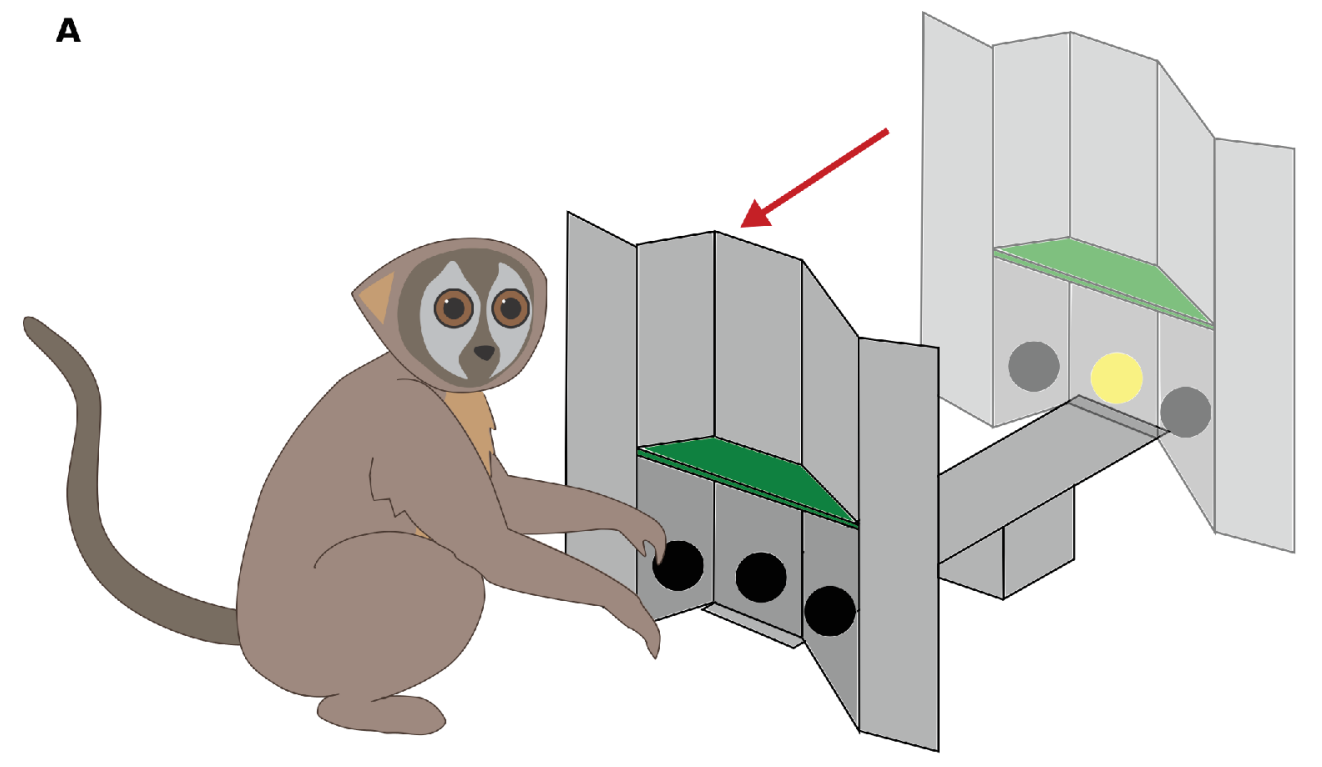

B

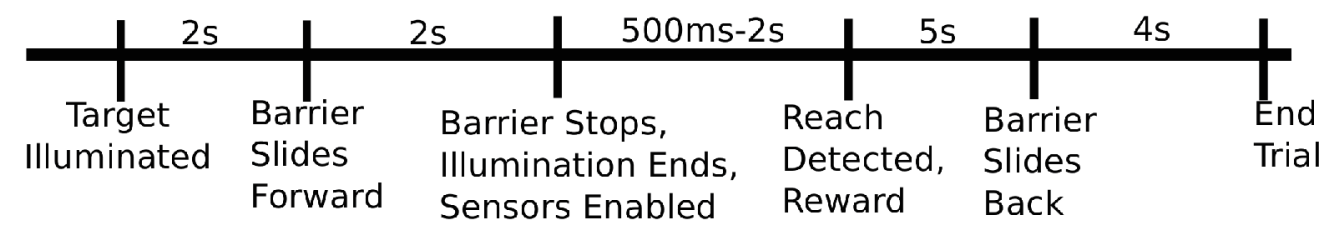

Figure 3. Experimental paradigm. (A) In the experiment, the monkey was seated facing metal barrier, with door 1 to its right. (B) Timeline of the visually-cued reach task (see Behavioral Task for details). 
The task of interest for our experiments was a visually-cued task (Figure 3). The experimental setup was housed in a sound-proof room, with very dim red light since the Aotus is nocturnal and falls asleep in bright lighting conditions. The monkeys were seated in a primate chair (Crist Instrument Co. polycarbonate primate chair for New World monkeys) facing front-center of the apparatus. The monkeys were restrained inside the chair with a soft, figure- 8 harness.

Each trial of the task started with the barrier away from the subjects. The control program randomly chose one of the three reward locations, and the corresponding LED lit up for 2 seconds. The door stayed illuminated for another 2 seconds as the slider brought the metal barrier forward into the monkey's reach, at which time the reach sensors became active. When the IR sensors detected a correct hand reach, a pellet was dispensed for the monkey into the corresponding trap. Five seconds after any reach detection, traps were activated to clear any pellet residues and the barrier slid back to the start position. A new trial then started after a 2 second inter-trial interval.

To prevent the monkey from developing a spatial preference towards a particular door, correction trials were imposed as trials following incorrect trials, with the target location unchanged. A treat was given for correct performance on correction trials, however, and an incorrect reach in a correction trial would results in no treat and another subsequent correction trial with the same target location.

\section{Results}

\subsection{Behavior}

Monkey A spent 20 sessions over 35 days getting used to the device following the procedures described above before starting the experimental task. Monkey P spent 23 sessions over 40 days before starting the experimental task.

Each visually-cued task session lasted between 60-90 minutes, depending on animal motivation. The total number of trials for each session was between 50-100, including error trials and correction trials. Monkey A performed five sessions of 100 trials each for this task, of which $78.6 \pm 7.6 \%$ (mean \pm standard deviation) were random target trials, and $21.4 \pm 7.6 \%$ were correction trials. The session performance, measured as the percentage of correct reaches toward random targets, increased over the sessions (Spearman rank correlation test, $\mathrm{p}=0.030$ ), eventually reaching 92.3\% accuracy (Figure 4 ). Monkey P performed 32 sessions of $81.4 \pm 19.7$ trials (mean \pm standard deviation) each for the task, of which $66.0 \pm 14.2 \%$ were random target trials, and $34.0 \pm 14.2 \%$ were correction trials. Its session performance increased over the sessions as well (Spearman rank correlation test, $p=4.09 \mathrm{e}-5$ ), reaching a maximum of $90.1 \%$ accuracy (Figure 4 ). These steady improvements to consistently high performance demonstrate that the monkeys have learned to recognize the association between the light stimulus and reward location.

In $85 \%$ of all trials, the time between the barrier stopping and the monkey's hand reaching the target was less than 2 seconds. The recorded videos showed that the preemptive reaching attempts accounted for most incorrect trials in the later sessions. The monkeys would sometimes reach preemptively for the door before the slider stopped and reach sensors activated. Upon receiving no reward, the monkey would reach for a different door. At this point the reach sensors were activated and consequently detected a wrong reach, resulting in an incorrect trial.

The device functioned reliably, with malfunctions associated with the dispenser mechanism occurring roughly once every 20 sessions. These were usually due to pellet breakage obstructing the horizontal slider or the vertical channel. The blockages were cleared easily by poking the slider channels with a thin wire. 


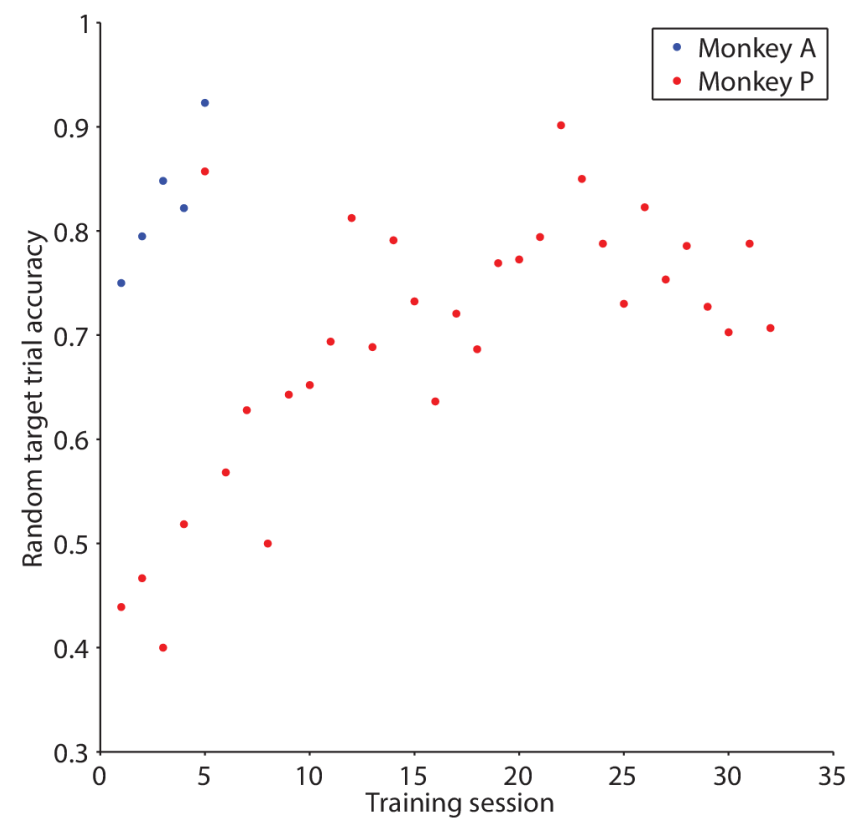

Figure 4. Random target reach performance in the visually-cued experimental reach task for the two monkeys.

\subsection{Neurophysiology}

Following initial behavioral training (Fig. 4), we started systematic neural recording in monkey A. Here we present neuronal data from four recording sessions. In one session, the behavioral task was the same as during training. In the following three sessions, the task modification was introduced: the light cue was turned on only after the slider stopped in front of the monkey. All the details of these neurophysiological data will be addressed in our future publications. Here we only report directional tuning properties of M1 neurons for the reach and grasp period of the task.

A

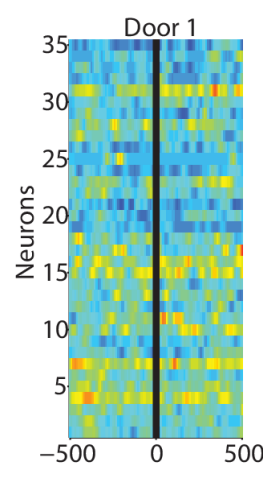

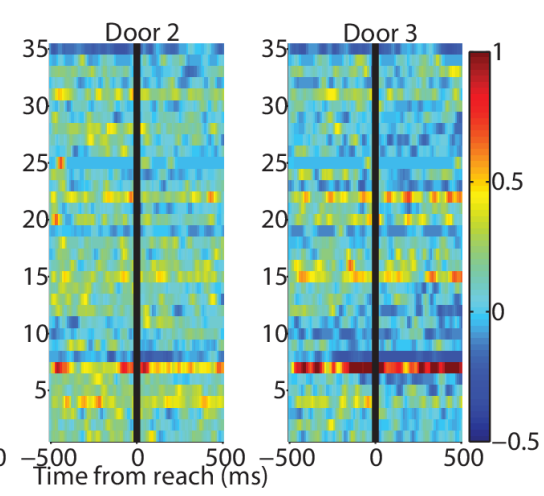

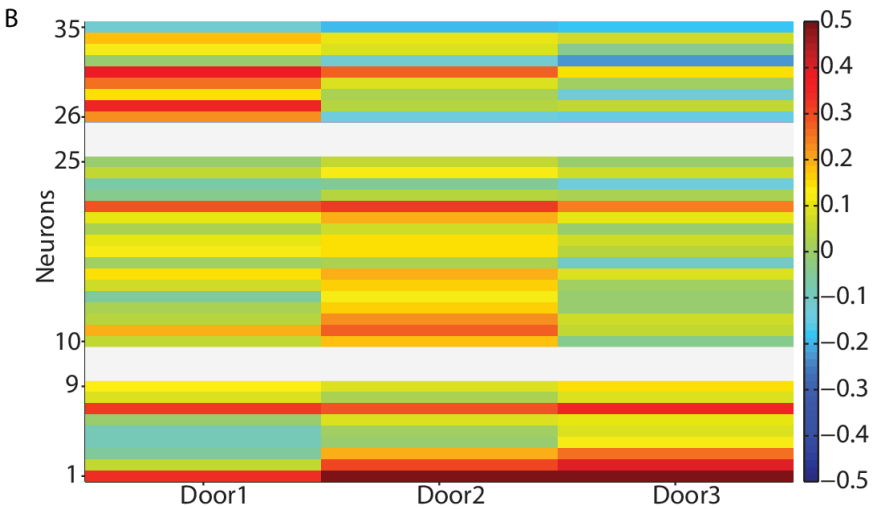

Figure 5. M1 Neurons. (A) PETHs for M1 neurons in Monkey A, averaged over all trials of the same door location in an example session. The vertical black lines indicate reach detection from the IR sensors. (B) Tuning of Monkey A's M1 neurons to the different door locations in the same session. 
Horizontal white lines separate neurons that prefer door 1, 2, and 3, respectively. Colors represent normalized firing rates in both plots.

The color plot of Figure 5a shows perievent time histograms (PETHs) for $35 \mathrm{M} 1$ neurons recorded in a representative session. In this plot, horizontal lines represent the trial average PETHs for each neuron. The PETHs were calculated by first dividing the entire session into $10 \mathrm{~ms}$ time-bins, which were used to determine the time-bin mean and standard deviations. Each time-bin was then normalized with those statistics to obtain normalized firing rates. The PETHs were centered on the time when the monkey's hand reached the target. Clearly, the neurons exhibited different patterns of activity when the monkey reached for different doors, i.e. they were directionally tuned (Georgopoulos, $\underline{\text { Schwartz et al. 1986). }}$.

To quantify directional tuning of each neuron, we calculated average firing rate for the interval 300 to $100 \mathrm{~ms}$ relative to the time of reaching the target. Average rates were calculated individually for each trial and then entered into Kruskal-Wallis analysis to assess statistical significance of directional tuning. On average, $47.9 \pm 6.3 \%$ (mean \pm standard error) of the M1 neurons were significantly tuned in the sessions analyzed.

Preferred direction was assessed as the target for which the firing rate was maximal for the analysis interval. Figure 5B shows neuronal preferred directions for all recorded neurons for the same session as in Fig. 5A. Figure 6 shows the average fraction of neurons by preferred direction. $42.1 \pm 5.5 \%$ of the M1 neurons preferred the contralateral target, $26.4 \pm 7.6 \%$ preferred the center target, and $31.4 \pm 2.6 \%$ preferred the ipsilateral target.

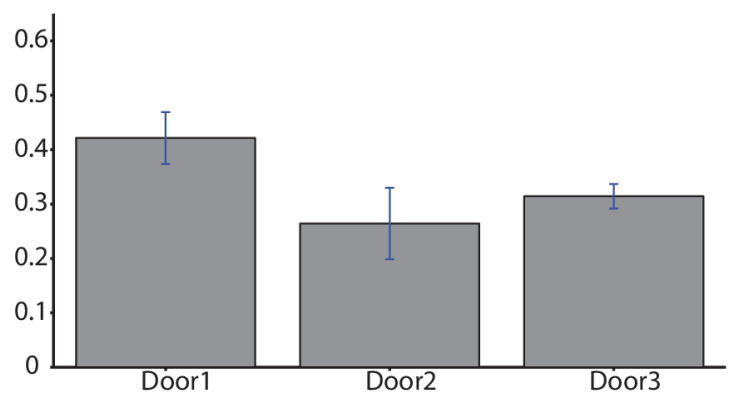

Figure 6. Percentage (mean and standard error) of Monkey A's recorded M1 neurons that are tuned to each door location, averaged over all sessions.

Thus, our experimental apparatus permitted automation of a reach-to-grasp task, and could be combined with neurophysiological recordings to assess the directional tuning properties in a population of cortical neurons.

\section{Discussion \& Conclusions}

This report describes a robust, portable, and pneumatically controlled apparatus integrating precise reward and stimulus delivery suitable for behavioral neurophysiology experiments in New World monkeys. The device was successful in training owl monkeys to perform a visually-cued reach task. The simple, gravity-fed pellet dispenser allowed for reliable and precise control of food delivery without using an elaborate measurement method (Pinkston, Ratzlaff et al. 2008). Further, the pneumatic actuation eliminated electrical noise while keeping the system easy to maintain.

Our entire apparatus can easily interface with a PC through multiple methods. This allows for 
more precise stimulus control, synchronization with other experimental devices, more accurate data collection, and for completely automated behavioral experiments. This could significantly improve neurophysiological and behavioral experiments that are manually administered. For example, in the reverse-contingency task in tamarinds and squirrel monkeys, respectively, the experimenters manually presented and dispensed different-sized rewards to the monkeys (Anderson, Awazu et al. 2000; Kralik, Hauser et al. 2002). During the experiment, the different time durations and reward locations were approximated. To minimize possible bias, the effects of different experimenters, their appearances, and where they looked during experiments had to be evaluated as well. Further, multiple researchers were needed to perform the experiment and record the responses at the same time. By modifying the presented apparatus to use transparent sliding doors, those experiments could be automated, and the extra procedures and approximations eliminated.

As pointed out in Mitz et al., 2001, the feeder designs for primates, New World monkeys or otherwise, need the ability to manipulate the visibility and availability of the reward. Our apparatus provides a number of ways to address these issues through the use of distance manipulation, traps, and sliding doors. The design is simple and flexible, and can be easily modified by swapping in transparent sliding doors and adding more valves to control the traps individually. Further, the design is modular such that a sliding barrier with more than three doors can be easily constructed for larger experiments.

Our set-up allowed the monkeys to use both hands in the arm-reaching experiment, but their choice of which hand to use can also be controlled. One method is to adapt the approach used by Takemi (Takemi, Kondo et al. 2014), where a transparent wall is erected in front of the monkey, and cut out holes on either the left or right side of the wall correspond to which hand the monkey is allowed to use for reaching.

While we only performed experiments with owl monkeys whose heads were not restrained, the apparatus should be able to adapt to head-fixed New World monkeys as well (Perryman, Lindsley et al. 1980; Bieser and Mueller-Preuss 1996). Perryman et al. demonstrated that head-fixed squirrel monkeys can achieve saccades up to 45 degrees to the right or left. Therefore, for head-fixed experiments, the angle between neighboring panels of the metal-barrier should be adjusted to fit within the head-fixed monkey's 90 degrees of vision. With current settings, when the metal-barrier is within the monkey's reach, each side door is exactly 45 degrees from the eyes, so only minor adjustments of the door placement will be needed for the fixed-head experiments.

Finally, the device's portability and compactness allow it to be modified to be used free-roaming primate experiments (Rolls, Robertson et al. 1997; Ludvig, Botero et al. 2001; Schwarz, Lebedev et al. 2014), where the monkey is not restrained in a primate chair. As most components of our device are built upon a single sliding metal barrier, it is possible to build a housing around the barrier to enclose the feeder and trap mechanisms. The metal-barrier can be reinforced and serve as a movable or fixed wall of the monkey's enclosure with minimal modifications. Specific types of monkey interactions with the apparatus would then be rewarded with food pellets. With wireless neural recordings and multiple devices, social interaction experiments such as those involving multiple monkeys reaching for the cued reward locations on separate devices can be designed. Such interactions may also be implemented as brain-to-brain interface experiments (Pais-Vieira, Lebedev et al. 2013; Pais-Vieira, Chiuffa et al. 2015; Ramakrishnan, Ifft et al. 2015).

Owl monkeys and other species of the New World monkeys are of great interest to neurophysiological and BMI research. They socially interact, vocalize, and communicate, and all these behaviors are of interests to neurophysiologists. In certain cases, New World monkeys can provide great alternatives to the more commonly-used rhesus monkeys in neurophysiological research. Due to these benefits, the New World monkeys as animal models have the potential to lead to important experimental results more easily. Our automated apparatus make controlled experiments with these animals even easier. 


\section{Acknowledgements}

This work was funded by the NIH Director's Pioneer Award DP1MH099903 to MALN; and an NSF graduate research fellowship to JA. The content is solely the responsibility of the authors and does not necessarily represent the official views of the Office of the NIH Director or the NIH.

\section{References Cited}

Abee, C. (1989). "The Squirrel Monkey in Biomedical Research." ILAR J 31: 11-20.

Allman, J. M. and J. H. Kaas (1971). "A representation of the visual field in the caudal third of the middle tempral gyrus of the owl monkey (Aotus trivirgatus)." Brain Res 31(1): 85-105.

Anderson, J. R., S. Awazu, et al. (2000). "Can squirrel monkeys (Saimiri sciureus) learn self-control? A study using food array selection tests and reverse-reward contingency." J Exp Psychol Anim Behav Process 26(1): 87-97.

Bao, S., E. F. Chang, et al. (2004). "Temporal plasticity in the primary auditory cortex induced by operant perceptual learning." Nat Neurosci 7(9): 974-981.

Batista, A. P., C. A. Buneo, et al. (1999). "Reach plans in eye-centered coordinates." Science 285(5425): 257-260.

Bieser, A., P. Muller-Preuss (1996). "Auditory responsive cortex in the squirrel monkey: neural responses to amplitude-modulated sounds.” Experimental Brain Research 108(2): 273-284.

Carmena, J. M., M. A. Lebedev, et al. (2003). "Learning to control a brain-machine interface for reaching and grasping by primates." PLoS Biol 1(2): E42.

Fitzsimmons, N., J. H. An, et al. (2009). Towards a Bi-Directional BrainMachine Interface: Simultaneous Microstimulation and Recording in Owl Monkeys. Society for Neuroscience Annual Meeting, Chicago, IL.

Fitzsimmons, N. A., W. Drake, et al. (2007). "Primate reaching cued by multichannel spatiotemporal cortical microstimulation." J Neurosci 27(21): 5593-5602.

Flash, T. and E. Henis (1991). "Arm trajectory modifications during reaching towards visual targets." 无 Cogn Neurosci 3(3): 220-230.

Georgopoulos, A. P., R. E. Kettner, et al. (1988). "Primate motor cortex and free arm movements to visual targets in three-dimensional space. II. Coding of the direction of movement by a neuronal population." J Neurosci 8(8): 2928-2937.

Georgopoulos, A. P., A. B. Schwartz, et al. (1986). "Neuronal population coding of movement direction." Science 233(4771): 1416-1419.

Ifft, P. J., S. Shokur, et al. (2013). "A brain-machine interface enables bimanual arm movements in 
monkeys." Sci Transl Med 5(210): 210 ra154.

Kaas, J. H. (1987). "The organization of neocortex in mammals: implications for theories of brain function." Annu Rev Psychol 38: 129-151.

Kettner, R. E., A. B. Schwartz, et al. (1988). "Primate motor cortex and free arm movements to visual targets in three-dimensional space. III. Positional gradients and population coding of movement direction from various movement origins." J Neurosci 8(8): 2938-2947.

Kralik, J. D., D. F. Dimitrov, et al. (2001). "Techniques for long-term multisite neuronal ensemble recordings in behaving animals." Methods 25(2): 121-150.

Kralik, J. D., M. D. Hauser, et al. (2002). "The relationship between problem solving and inhibitory control: cotton-top tamarin (Saguinus oedipus) performance on a reversed contingency task." J Comp Psychol 116(1): 39-50.

Lacquaniti, F. and R. Caminiti (1998). "Visuo-motor transformations for arm reaching." Eur J Neurosci 10(1): 195-203.

Lebedev, M. A. and M. A. Nicolelis (2006). "Brain-machine interfaces: past, present and future." Trends Neurosci 29(9): 536-546.

Lebedev, M. A. and S. P. Wise (2001). "Tuning for the orientation of spatial attention in dorsal premotor cortex." Eur J Neurosci 13(5): 1002-1008.

Livesey, P. J., H. Lowe, et al. (1972). "Automated Apparatus for Study of Learning in Monkey and Rat." Australian Journal of Psychology 24(2): 211-\&.

Ludvig, N., J. M. Botero, et al. (2001). "Single-cell recording from the brain of freely moving monkeys." J Neurosci Methods 106(2): 179-187.

Mansfield, K. (2003). "Marmoset models commonly used in biomedical research." Comp Med 53(4): 383-392.

McIntyre, J., F. Stratta, et al. (1998). "Short-term memory for reaching to visual targets: psychophysical evidence for body-centered reference frames." J Neurosci 18(20): 8423-8435.

Merzenich, M. M., J. H. Kaas, et al. (1983). "Progression of change following median nerve section in the cortical representation of the hand in areas $3 \mathrm{~b}$ and 1 in adult owl and squirrel monkeys." Neuroscience 10(3): 639-665.

Mitz, A. R. (2005). "A liquid-delivery device that provides precise reward control for neurophysiological and behavioral experiments." J Neurosci Methods 148(1): 19-25.

Mitz, A. R., S. A. Boring, et al. (2001). "A novel food-delivery device for neurophysiological and neuropsychological studies in monkeys." J Neurosci Methods 109(2): 129-135.

Nicolelis, M. A., A. A. Ghazanfar, et al. (1998). "Simultaneous encoding of tactile information by three primate cortical areas." Nat Neurosci 1(7): 621-630.

Nudo, R. J. and G. W. Milliken (1996). "Reorganization of movement representations in primary motor 
cortex following focal ischemic infarcts in adult squirrel monkeys." J Neurophysiol 75(5): 2144-2149.

Nudo, R. J., G. W. Milliken, et al. (1996). "Use-dependent alterations of movement representations in primary motor cortex of adult squirrel monkeys." J Neurosci 16(2): 785-807.

O'Doherty, J. E., M. A. Lebedev, et al. (2011). "Active tactile exploration using a brain-machine-brain interface." Nature 479(7372): 228-231.

Pais-Vieira, M., G. Chiuffa, et al. (2015). "Building an organic computing device with multiple interconnected brains." Sci Rep 5: 11869.

Pais-Vieira, M., M. Lebedev, et al. (2013). "A brain-to-brain interface for real-time sharing of sensorimotor information." Sci Rep 3: 1319.

Perryman, K. M., D. F. Lindsley, et al. (1980). "Pulvinar neuron responses to spontaneous and trained eye movements and to light flashes in squirrel monkeys.” Electroencephalography and Clinical Neurophysiology 49(1): 152-161.

Pinkston, J. W., K. L. Ratzlaff, et al. (2008). "An inexpensive infrared detector to verify the delivery of food pellets." J Exp Anal Behav 90(2): 249-255.

Ramakrishnan, A., P. J. Ifft, et al. (2015). "Computing Arm Movements with a Monkey Brainet." $\underline{\text { Sci }}$ Rep 5: 10767.

Rolls, E. T., R. G. Robertson, et al. (1997). "Spatial view cells in the primate hippocampus." Eur J Neurosci 9(8): 1789-1794.

Sandler, A. (2005). Neuronal correlates of somatosensorimotor learning and decision making in primates. $\mathrm{PhD}$, Duke University.

Scherberger, H. and R. A. Andersen (2007). "Target selection signals for arm reaching in the posterior parietal cortex." J Neurosci 27(8): 2001-2012.

Scherberger, H., M. A. Goodale, et al. (2003). "Target selection for reaching and saccades share a similar behavioral reference frame in the macaque." J Neurophysiol 89(3): 1456-1466.

Schwartz, A. B., R. E. Kettner, et al. (1988). "Primate motor cortex and free arm movements to visual targets in three-dimensional space. I. Relations between single cell discharge and direction of movement." J Neurosci 8(8): 2913-2927.

Schwarz, D. A., M. A. Lebedev, et al. (2014). "Chronic, wireless recordings of large-scale brain activity in freely moving rhesus monkeys." Nat Methods 11(6): 670-676.

Takemi, M., T. Kondo, et al. (2014). "Three-dimensional motion analysis of arm-reaching movements in healthy and hemispinalized common marmosets." Behavioural Brain Research 275: 259-268.

Takemoto, A., A. Izumi, et al. (2011). "Development of a compact and general-purpose experimental apparatus with a touch-sensitive screen for use in evaluating cognitive functions in common marmosets." J Neurosci Methods 199(1): 82-86. 
Wessberg, J., C. R. Stambaugh, et al. (2000). "Real-time prediction of hand trajectory by ensembles of cortical neurons in primates." Nature 408(6810): 361-365.

Wilson, F. A., B. H. Kim, et al. (2005). "An automated food delivery system for behavioral and neurophysiological studies of learning and memory in freely moving monkeys." Behav Res Methods 37(2): 368-372.

Wise, S. P., G. Di Pellegrino, et al. (1992). "Primate premotor cortex: dissociation of visuomotor from sensory signals." J Neurophysiol 68(3): 969-972. 
Filename: Yin_JNeurosciMethods_RevManuscript

Directory: D: $\quad$ C:bertlieee

Template: $\quad$ C: UUsers $\quad$ abalolong.SPI-

GLOBAL\AppData\Roaming \Microsoft $\backslash$ Templates $\backslash$ Normal.dotm

Title:

Subject:

Author: Susan Halkiotis

Keywords:

Comments:

Creation Date: $\quad$ 8/12/2016 10:36:00 AM

Change Number: 2

Last Saved On: $\quad$ 8/12/2016 10:36:00 AM

Last Saved By: Balolong, Ariston

Total Editing Time: 0 Minutes

Last Printed On: $\quad$ 8/12/2016 10:37:00 AM

As of Last Complete Printing

Number of Pages: 15

Number of Words: $\quad$ 6,264 (approx.)

Number of Characters: 33,889 (approx.) 